Methods: there were examined 22 children at the age of 7 to 17 years old diagnosed with CAPS-9, TRAPS-8, FMF-5. Among them there were 12 boys and 10 girls. The diagnosis in all the patients was confirmed through detection of pathogenic mutations in the NLRP3, TNFRSF1A and MEFV genes. The following methods were used: a clinical conversation; memory diagnostics (learning by heart of 10 words, a pictogram using cues taking into account the patients' age); attention diagnostics (Schulte tables); thinking diagnostics (establishing a sequence of events, "four is a crwod", simple analogies, interpretation of proverbs); emotional and communicative fields (the Eight-Color Luscher Test; CMAS (adaptation by A. Prikhozhan); STAI test, a drawing called "an animal that does not exist" and "a house-a tree-a man").

Results: The memory study revealed in all patients with TRAPS and FMF high and medium values of short-term and long-term memory, in patients with CAPS - a low level of short-term auditory-speech memory, information storage and indirect memorization in $1 / 3$ of patients. In $100 \%$ of the examined patients with TRAPS, a significant decrease in all processes of attention and distribution of attention. In 1/3 of patients with CAPS, an increased exhaustion of attention was registered and in $11 \%$ - a decrease in its stability. In patients with FMF, attention disorders were not detected. In $44 \%$ of patients with CAPS, a decrease in the level of generalization and difficulties in establishing causal relationships were registered. In $25 \%$ of patients with TRAPS a decrease in the level of generalization, in $12.5 \%$ - difficulties in establishing cause-effect relationships, inertia of thinking in $37.5 \%$. In $60 \%$ of patients with FMF: a decrease in the level of generalization, in $80 \%$ : difficulties in establishing cause-effect relationships, inertia of thinking in $20 \%$. In the emotional sphere, patients with CAPS, TRAPS, and FMF demonstrated signs of aggression ( $11.1 \%, 20 \%$ and $20 \%$ of patients, respectively), communicative disorders $(77.8 \%-80 \%-80 \%)$, and reduced social adaptation $(55.5 \%$ - $80 \%-80 \%)$, a tendency to form neurotic fears (22\% - 40\% - 40\%). A high level of personal anxiety was noted in $1 / 3$ of patients with CAPS and $40 \%$ of patients with FMF.

Conclusion: various psychological disorders in the cognitive and emotional fields were noted in the majority of the examined patients with monogenic auto-inflammatory diseases. In patients with TRAPS, attention processes are most significantly affected; in patients with CAPS, memory is more often affected. In patients with FMF, disorders in thinking processes are revealed more often. In the emotional sphere, most patients with all the three forms of AID note communicative disorders and social adaptation.

Disclosure of Interests: None declared

DOI: 10.1136/annrheumdis-2020-eular.4182

\section{FRI0633-HPR \\ PHYSICAL AND SOCIAL ACTIVITY OF PATIENTS SUFFERING FROM JUVENILE IDIOPATHIC ARTHRITIS}

\section{T. Shelepina1}

${ }^{1}$ V.A.Nasonova Research Institute of Rheumatology, Pediatric, Moscow, Russian Federation

Background: Juvenile Idiopathic Artritis (JIA) is a chronic, disabling disease of a childhood age that significantly limits the patient's capabilities and affects the life quality. Modern drug therapies can prevent most of the negative consequences of the disease and maintain satisfactory functional abilities of patients.

Objectives: to examine the nature of the daily physical and social activities of patients suffering from JIA.

Methods: the study included 236 patients aged from 4 to 17 years old undergoing in-patient treatment. Among them, $146(62 \%)$ - polyarthritis and spreading oligoarthritis, including $13(5.5 \%)$ - with damage to the eyes, persistent oligoarthritis - $60(25 \%)$, including $26(11 \%)$ with damage to the eyes, systemic JIA 22 (16\%), enthesitis-associated $8(3 \%)$. The patients were divided into the following age groups: pre-school age (4-6 years old) -26 patients (11\%), primary school age (7-10 years old) - 54 patients (23\%); an average school age (11-12 years old) -49 patients $(21 \%)$, senior school age (13-15 years old) -52 patients $(22 \%)$, youth (16-17 years old) -55 patients (23\%). The assessment methods: collection of their pharmacological history, questioning (with an author's questionnaire) the parents of children aged from 4 to 10 years and older patients themselves, VAS pain evaluation, a $\mathrm{CHAQ}$ questionnaire.

Results: 10 (4\%) patients received NSAID, 88 (38\%) patients - synthetic basic anti-inflammatory drugs, biological therapy - $133(56 \%), 5$ patients $(2 \%)$ did not receive any drug therapy. An average value of the VAS pain evaluation: $2.5 \mathrm{~cm}$, the CHAQ functional insufficiency is low: 137 patients (58\%), medium insufficiency in 88 patients $(37 \%)$, a severe one in 11 patients $(5 \%)$. Children's educational institutions were regularly visited by 199 (85\%) patients, leisure activities were enjoyed by 90 (38\%) patients; at that, the highest rate of leisure activities was recorded for the age group $112-12$ years ( $67 \%$ of the entire group), daily walks were recorded in $172(73 \%)$ patients, doing homework on a regular basis by $155(66 \%)$ patients, regular doing exercises of therapeutic gymnastics recorded in $55(24 \%)$ patients. Conclusion: The social activity of patients suffering from JIA can be estimated as satisfactory, while their physical activity as inadequate. An adequate social and physical activation of patients with JIA being in the remission status is required.
This can be facilitated by educational programs for patients and their parents, a joint discussion of issues on the social and physical activity of patients and their parents with rheumatologists and rehabilitation therapy specialists.

Acknowledgments: I thank senior researcher, E. S. Fedorov MD for his help in completing the work and preparing abstracts

Disclosure of Interests: None declared

DOI: 10.1136/annrheumdis-2020-eular.4208

\section{FRI0634-HPR LEVEL OF PHYSICAL ACTIVITY IN ANTIPHOSPHOLIPID SYNDROME AND ITS RELATIONSHIP TO ATHEROSCLEROSIS PROGRESSION - ANALYSIS OF THE SERBIAN COHORT}

A. Djokovic ${ }^{1,2}$, L. Stojanovich $^{3}$, N. Stanisavljevic ${ }^{4}$, G. Bogdanovic ${ }^{5}$, S. Djokic ${ }^{6}$ ${ }^{1}$ University Clinical Hospital Center Bezanijska kosa, Cardiology, Belgrade, Serbia; ${ }^{2}$ Faculty of Medicine, University of Belgrade, Belgrade, Serbia; ${ }^{3}$ University Clinical Hospital Center Bezanijska kosa, Rheumatology, Belgrade, Serbia; ${ }^{4}$ University Clinical Hospital Center Bezanijska kosa, Hematology, Belgrade, Serbia; ${ }^{5}$ University Clinical Hospital Center Bezanijska kosa, Physical Medicine and Rehabilitation, Belgrade, Serbia; ${ }^{6}$ Clinical Center of Serbia, Clinic of Pulmonology, Belgrade, Serbia

Background: Systemic lupus erythematosus (SLE) and antiphospholipid syndrome (APS) are associated with an increased risk of developing cardiovascular diseases as a result of complex interaction between traditional risk factors, chronic inflammation and specific impact of antibodies on endothelium. There are very limited data regarding level of physical activity (PA) in APS patients.

Objectives: To analyze different domains of PA in Serbian APS patients and their possible relationship to clinical and laboratory criteria of the main disease.

Methods: From a large Serbian APS database comprehending 527 APS patients (371 Primary - PAPS, and 156 APS associated with other autoimmune diseases predominantly systemic lupus erythematosus (SLE)) we interviewed 51 APS patients, age range of 15-69 years: 29 patients with primary APS (PAPS), 25 women, 4 men, age 44 \pm 11.50 , and 22 APS/SLE, 18 women, 4 men, age 48.41 \pm 11.75 , using a long form of The International Physical Activity Questionnaire (IPAQ), translated onto Serbian language. Data on last seven days of PA divided onto leisure time PA, domestic and gardening (yard) activities, work-related PA and transport-related PA were acquired, and proposed scoring method was used. Based on the level of PA, patients were categorized to low, moderate or high level of PA. For the purpose of insight into atherosclerotic progression, we performed color Doppler scan of carotid arteries in all patients and presence of atherosclerotic plaques has been notified. Results: Average total PA score was $7706.18 \pm 11771.97$ MET-minutes/week. The greatest average values for different PA domains were for work $(2733.21 \pm 6158.66$ MET-minutes/week) and domestic/garden/yard (2522.31 +3847.24 MET-minutes/ week) and the lowest scores achieved in leisure time $(500.87 \pm 695.45$ MET-minutes/week). Majority of Serbian APS patients had low or moderate level of PA (37.3\%, 43.1\%, respectively) whereas lowest percentage was in high category of PA (19.6\%). All domains of PA were significantly negatively correlated to age and BMI. There were no significant difference regarding PA scores between PAPS and APS/SLE patients. Although higher percentage of PAPS patients had high level of PA (27.65 compared to $9.1 \%$ of SLE/APS), the overall difference was not significant. There was no significant difference regarding antiphospholipid antibody (aPL) type or thrombotic/obstetric events presence. Significant difference occurred regarding presence of carotid arteries plaques. APS patients with lower PA scores had significantly higher prevalence of carotid arteries plaque especially for PA in transportation ( $p=0.004)$, and total PA $(p=0.025)$

Conclusion: Serbian APS patients at younger age, tend to have low or moderate level of PA, with the lowest level of activity in leisure time. Low level of PA was undoubtedly related to progression of atherosclerosis in these patients, emphasizing a need for PA promotion in APS.

Disclosure of Interests: Aleksandra Djokovic Speakers bureau: KRKA, Astra Zeneca, Actavis, Ljudmila Stojanovich: None declared, Natasa Stanisavljevic: None declared, Gordana Bogdanovic: None declared, Sandra Djokic: None declared

DOI: 10.1136/annrheumdis-2020-eular.2548

\section{FRI0635-HPR PATIENTS' MOTIVATION AND GOALS FOR THUMB CARPOMETACARPAL OSTEOARTHRITIS SURGERY}

E. M. H. Gravås ${ }^{1}$, I. Kjeken ${ }^{1}$, R. Nossum ${ }^{2}$, R. E. Mehl Eide ${ }^{3}$, Å. Klokkeide ${ }^{4}$ K. Hoegh Matre ${ }^{3}$, M. Olsen ${ }^{4}, \varnothing$. Andreassen ${ }^{1}$, N. Osteras ${ }^{1}$, A. T. Tveter ${ }^{1}$. ${ }^{1}$ Diakonhjemmet Hospital, Department of Rheumatology, National Advisory Unit on Rehabilitation in Rheumatology, Oslo, Norway; ${ }^{2}$ Trondheim University Hospital, St. Olavs Hospital, Trondheim, Norway; ${ }^{3}$ Haukeland University Hospital, Bergen, Norway; ${ }^{4}$ Haugesund Rheumatism Hospital AS, Haugesund, Norway

Background: Osteoarthritis (OA) in the thumb carpometacarpal joint (CMCJ) is a prevalent disease which may lead to structural damage, severe pain and 